\title{
Current non-conservation effects in ultra-high energy neutrino interactions
}

\author{
R. Fiore ${ }^{1 \dagger}$ and V.R. Zoller ${ }^{2 \ddagger}$ \\ ${ }^{1}$ Dipartimento di Fisica, Università della Calabria \\ and \\ Istituto Nazionale di Fisica Nucleare, Gruppo collegato di Cosenza, \\ I-87036 Rende, Cosenza, Italy \\ ${ }^{2}$ ITEP, Moscow 117218, Russia
}

\begin{abstract}
The overall hardness scale of the ultra-high energy neutrino-nucleon interactions is usually estimated as $Q^{2} \sim m_{W}^{2}$. The effect of non-conservation of weak currents pushes this scale up to the top quark mass squared and changes dynamics of the scattering process. The Double Leading Log Approximation provides simple and numerically accurate formula for the top-bottom contribution to the total cross section $\sigma^{\nu N}$. Corresponding correction to $\sigma^{\nu N}$ appears to be numerically large. It is comparable with the leading contribution evaluated in the massless quark approximation.
\end{abstract}

†email address: fiore@cs.infn.it

‡email address: zoller@itep.ru 
New ideas [1] about the origin of neutrino fluxes from active galactic nuclei, gamma ray bursts or from decay of exotic heavy particles inspired many publications on the ultra-high energy (UHE) neutrino-nucleon total cross sections $\sigma^{\nu N}[2,3,4,5]$. The UHE interactions correspond to neutrino energy above $E_{\nu} \sim 10^{8} \mathrm{GeV}$, where the gauge boson exchange probes the gluon density in the target nucleon at very small values of Bjorken $x$. The gluon density at small $x$ is known to be a rapidly rising function of $Q^{2}$. Its rise is tamed, however, by the propagator of the gauge boson which sets the restriction $[2,3,4,5]$

$$
Q^{2} \lesssim m_{W}^{2}
$$

This value of $Q^{2}$ represents the overall hardness scale of the process induced by the light quark current, $m_{q}^{2} \ll Q^{2}$. The top-bottom current needs special care. In this communication we show that the charged current non-conservation (CCNC) effect pushes the hardness scale up to the top quark mass squared, $m_{t}^{2}$, and crucially changes dynamics of the process ${ }^{1}$.

The differential cross section for the neutrino-nucleon interactions is expressible in terms of the longitudinal, $F_{L}$, transverse, $F_{T}$, and left-right antisymmetric, $F_{3}$, structure functions. In standard notations it reads

$$
x \frac{d \sigma^{\nu N}}{d x d Q^{2}}=\frac{G_{F}^{2}}{2 \pi}\left(\frac{m_{W}^{2}}{m_{W}^{2}+Q^{2}}\right)^{2}\left[(1-y) F_{L}+\left(1-y+\frac{y^{2}}{2}\right) F_{T}+y\left(1-\frac{y}{2}\right) x F_{3}\right],
$$

It is the longitudinal structure function $F_{L}$ which is the carrier of the CCNC effect. Indeed, for longitudinal/scalar W-boson with polarization vector $\varepsilon_{\mu}^{L}$ the vector or axial-vector transition vertex $W \rightarrow t \bar{b}$ is $\propto \varepsilon_{\mu}^{L} J_{\mu} \propto \partial_{\mu} J_{\mu} \propto m_{t} \pm m_{b}$. Therefore, $F_{L}$ which is $\propto \varepsilon_{\mu}^{L} T_{\mu \nu} \varepsilon_{\nu}^{L}$ provides a measure of the CCNC effect, here $T_{\mu \nu}$ stands for the imaginary part of the forward scattering Compton amplitude.

To the Leading $\log (1 / x)$ Approximation, the operational definition of the differential glue for any target is provided by the $\boldsymbol{\kappa}$-factorization representation ${ }^{2}$ corresponding to the gauge invariant sum of diagrams like that shown in Fig. 1

$$
\frac{d \sigma_{L}\left(x, Q^{2}\right)}{d z d^{2} \mathbf{k}}=\frac{\alpha_{W}}{\pi} \int \frac{d^{2} \boldsymbol{\kappa}}{\boldsymbol{\kappa}^{4}} \alpha_{S}\left(q^{2}\right) \mathcal{F}\left(x, \boldsymbol{\kappa}^{2}\right)\left(V_{S}+A_{S}+V_{P}+A_{P}\right)
$$

\footnotetext{
${ }^{1}$ Preliminary results have been reported at the Diffraction 2010 Workshop [6]

${ }^{2}$ The differential cross section $d \sigma_{L, T} / d z d^{2} \mathbf{k}$ to the lowest order in pQCD has been derived in [7]
} 


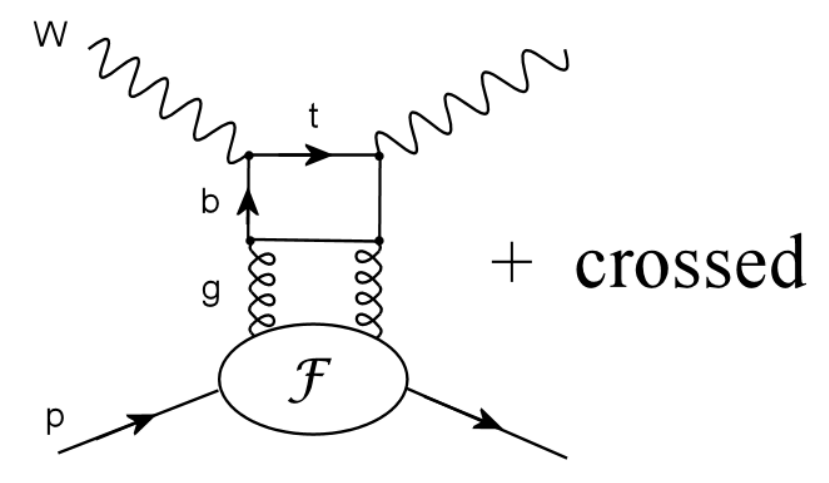

Figure 1: Diagrammatic representation of the $\boldsymbol{\kappa}$-factorization formula (3)

where $\alpha_{W}=g^{2} / 4 \pi, g^{2}=G_{F} m_{W}^{2} / \sqrt{2}$ and $\mathcal{F}$ stands for the differential gluon density

$$
\mathcal{F}\left(x, \boldsymbol{\kappa}^{2}\right)=\frac{\partial G\left(x, \boldsymbol{\kappa}^{2}\right)}{\partial \log \kappa^{2}} .
$$

We denoted by $\boldsymbol{\kappa}$ and $\mathbf{k}$ the gluon and $t$-quark transverse momenta, respectively, and by $z$ the fraction of the light-cone momentum of the $W$ carried by the top quark.

At small $x$ it is legitimate to discuss the $\nu N$-scattering in the laboratory frame in terms of interactions with the target of the $q \bar{q}$-pair which the light-cone W-boson transforms into at large upstream distances. The axial-vector $A_{S}$ and vector term $V_{S}$ describe the interaction with the target of the quark-antiquark $|t \bar{b}\rangle$ state with the angular momentum $L=0$ (S-wave),

$$
\begin{array}{r}
V_{S}\left(m_{t}, m_{b}\right)=\frac{g_{V}^{2}}{Q^{2}}\left\{2 Q^{2} z(1-z)+\right. \\
\left.\left(m_{t}-m_{b}\right)\left[(1-z) m_{t}-z m_{b}\right]\right\}^{2} \\
\times\left(\frac{1}{\mathbf{k}^{2}+\varepsilon^{2}}-\frac{1}{(\mathbf{k}-\boldsymbol{\kappa})^{2}+\varepsilon^{2}}\right)^{2}, \\
A_{S}\left(m_{t}, m_{b}\right)=\frac{g_{A}^{2}}{g_{V}^{2}} V_{S}\left(m_{t},-m_{b}\right),
\end{array}
$$

where

$$
\varepsilon^{2}=z(1-z) Q^{2}+(1-z) m_{t}^{2}+z m_{b}^{2}
$$

In the charged current neutrino interactions $g_{A}=-g_{V}=-1$ and $m_{b}$ and $m_{t}$ stand for the bottom and the top quark masses. The two terms $A_{P}$ and $V_{P}$ correspond to the quarkantiquark states with the angular momentum $L=1$ (P-wave) and are given by

$$
V_{P}\left(m_{t}, m_{b}\right)=\frac{g_{V}^{2}}{Q^{2}}\left(m_{t}-m_{b}\right)^{2}\left(\frac{\mathbf{k}}{\mathbf{k}^{2}+\varepsilon^{2}}-\frac{\mathbf{k}-\boldsymbol{\kappa}}{(\mathbf{k}-\boldsymbol{\kappa})^{2}+\varepsilon^{2}}\right)^{2}
$$




$$
A_{P}\left(m_{t}, m_{b}\right)=\frac{g_{A}^{2}}{g_{V}^{2}} V_{P}\left(m_{t},-m_{b}\right) .
$$

The P-wave component of the light-cone Fock state expansion for the longitudinal/scalar W-boson arises entirely due to the current non-conservation. In the P-wave quark-antiquark state $\left|q \bar{q}^{\prime}\right\rangle$ either quark or antiquark has wrong helicity, the quark is right-handed or antiquark is left-handed. Normally, this configuration is suppressed as $m_{q}^{2} / Q^{2}$ but in our specific case $Q^{2}$ is limited (see Eq.(1)) and $m_{q}^{2}=m_{t}^{2} \gg Q^{2}$. Therefore, to describe correctly the suppression of wrong helicity states one needs more accurate treatment. We addressed this issue in [8, 9] and quantified the CCNC effect in terms of the light cone wave functions in the color dipole basis. Here we reproduce and extend the results of $[8,9]$ making use of the momentum representation.

Consider first the P-wave component of Eq.(3). Separate the $\boldsymbol{\kappa}^{2}$-integration in (3) into the soft,

$$
\boldsymbol{\kappa}^{2} \ll \overline{k^{2}} \equiv \varepsilon^{2}+\boldsymbol{k}^{2}
$$

and hard,

$$
\kappa^{2} \gtrsim \overline{k^{2}}
$$

regions of the gluon momentum. For soft gluons upon the azimuthal integration we get

$$
\int d \varphi\left(\frac{\mathbf{k}}{\mathbf{k}^{2}+\varepsilon^{2}}-\frac{\mathbf{k}-\boldsymbol{\kappa}}{(\mathbf{k}-\boldsymbol{\kappa})^{2}+\varepsilon^{2}}\right)^{2} \simeq 2 \pi \boldsymbol{\kappa}^{2} \frac{\varepsilon^{4}+\left(\mathbf{k}^{2}\right)^{2}}{\left(\mathbf{k}^{2}+\varepsilon^{2}\right)^{4}} .
$$

In Eq.(3) the QCD running coupling $\alpha_{S}\left(q^{2}\right)$ enters the integrand at the largest relevant virtuality, $q^{2}=\max \left\{\overline{k^{2}}, \kappa^{2}\right\}$. To the Double Leading Log Approximation (DLLA) [10] one can take $q^{2}=\overline{k^{2}}$ and for soft gluons we arrive at the fully differential distribution of the t-quark in $z$ and $\mathbf{k}$,

$$
\frac{d \sigma_{L}^{P}\left(x, Q^{2}\right)}{d z d^{2} \mathbf{k}}=\alpha_{W}\left(g_{A}^{2}+g_{V}^{2}\right) \frac{m_{t}^{2}}{Q^{2}} \alpha_{S}\left(\overline{k^{2}}\right) G\left(x, \overline{k^{2}}\right) \frac{\varepsilon^{4}+\left(\mathbf{k}^{2}\right)^{2}}{\left(\mathbf{k}^{2}+\varepsilon^{2}\right)^{4}}
$$

where $m_{b}^{2}$ is neglected compared to $m_{t}^{2}$. Then, going from $d \sigma_{L} / d z d^{2} \mathbf{k}$ to the longitudinal structure function,

$$
F_{L}\left(x, Q^{2}\right)=\frac{Q^{2}}{4 \pi^{2} \alpha_{W}} \sigma_{L}\left(x, Q^{2}\right)
$$

we find the soft gluon contribution to the $\mathrm{P}$-wave component of $F_{L}$ 


$$
F_{L}^{P}\left(x, Q^{2}\right) \simeq \frac{m_{t}^{2}}{2 \pi^{2}} \int_{0}^{1} d z \int d^{2} \mathbf{k} \alpha_{S}\left(\overline{k^{2}}\right) G\left(x, \overline{k^{2}}\right) \frac{\varepsilon^{4}+\left(\mathbf{k}^{2}\right)^{2}}{\left(\mathbf{k}^{2}+\varepsilon^{2}\right)^{4}}
$$

where

$$
x=\frac{Q^{2}+M^{2}}{W^{2}+Q^{2}}
$$

and in the soft gluon approximation

$$
M^{2}=\frac{m_{t}^{2}+\mathbf{k}^{2}}{z}+\frac{m_{b}^{2}+\mathbf{k}^{2}}{1-z}
$$

The full $z$ integration can be separated into two domains $z_{m}<z<1$ and $0<z<z_{m}$, where

$$
z_{m}=\max \left\{0,\left[1-\left(m_{t}^{2}-m_{b}^{2}\right) / Q^{2}\right] / 2\right\}
$$

The leading contribution to $F_{L}^{P}$ comes from

$$
z \sim 1-\frac{m_{b}^{2}}{m_{t}^{2}+Q^{2}}
$$

when the t-quark carries almost 100 per cent of the longitudinal W's momentum [8], so that for $z_{m}<z<1$ we can make a substitution $d \varepsilon^{2}=-\left(Q^{2}+m_{t}^{2}\right) d z$. Then

$$
F_{L}^{P}\left(x, Q^{2}\right) \simeq \frac{m_{t}^{2}}{m_{t}^{2}+Q^{2}} \int_{m_{b}^{2}}^{\varepsilon_{m}^{2}} \frac{d \varepsilon^{2}}{\varepsilon^{2}} \frac{\alpha_{S}\left(\varepsilon^{2}\right)}{3 \pi} G\left(x, \varepsilon^{2}\right) .
$$

Here the hardness scale for $Q^{2} \leq m_{t}^{2}-m_{b}^{2}$ is

$$
\varepsilon_{m}^{2}=m_{t}^{2}
$$

and for higher $Q^{2}>m_{t}^{2}-m_{b}^{2}$ it is

$$
\varepsilon_{m}^{2}=\frac{1}{4}\left(Q^{2}+m_{t}^{2}+m_{b}^{2}\right)\left[1+\left(m_{t}^{2}-m_{b}^{2}\right) / Q^{2}\right]
$$

The CCNC also affects the S-wave component of the longitudinal structure function,

$$
F_{L}=F_{L}^{P}+F_{L}^{S}
$$

One can see that both the S-wave and P-wave parts of $F_{L}$ correspond to very different $z$-distributions. The $\mathrm{P}$-wave component is dominated by $z \sim 1$ while the S-wave term integrated over $\mathbf{k}$ has approximately uniform $z$-distribution. A narrow peak in the S-wave 
$z$-distribution at $z \rightarrow 1$ rises to $\sim\left(m_{t}^{2}+Q^{2}\right) / m_{b}^{2}$ but its width is $\delta z \sim m_{b}^{2} /\left(m_{t}^{2}+Q^{2}\right)$ and this singularity does not affect the DLLA estimate

$$
F_{L}^{S}\left(x, Q^{2}\right) \simeq \frac{2 \alpha_{S}\left(\overline{\varepsilon^{2}}\right)}{3 \pi} G\left(x, \overline{\varepsilon^{2}}\right)
$$

where

$$
\overline{\varepsilon^{2}} \simeq\left(Q^{2}+2 m_{t}^{2}\right) / 4
$$

We neglected here the contribution of hard gluons to the proton longitudinal structure function. Therefore, the DLLA gives the lower estimate for $F_{L}$.

Then the contribution to $\sigma^{\nu N}$,

$$
\sigma^{\nu N}=\int_{Q_{0}^{2}}^{s} d Q^{2} \int_{x_{t}}^{1} d x\left(\frac{d \sigma}{d x d Q^{2}}\right)
$$

coming from the absorption of longitudinal W-bosons (we call it the CCNC contribution) can easily be estimated, in Eq.(18) $x_{t}=\left(m_{t}^{2}+Q^{2}\right) / s, y=\left(m_{t}^{2}+Q^{2}\right) / x s$ and $s=2 m_{N} E_{\nu}$. At $E_{\nu}=10^{12} \mathrm{GeV}$ and for the input gluon density $G\left(x, k^{2}\right)$ specified in [11] this contribution appears to be equal to

$$
\sigma_{C C N C}^{\nu N} \simeq 0.45 \times 10^{-31} \mathrm{~cm}^{2}
$$

While for the gluon density from [12] we arrive at

$$
\sigma_{C C N C}^{\nu N} \simeq 0.56 \times 10^{-31} \mathrm{~cm}^{2}
$$

For comparison, the frequently used approximation of massless quarks gives the total cross section $\sigma^{\nu N}$ which for different input gluon densities varies in a rather wide range [4]. For example, at $E_{\nu}=10^{12} \mathrm{GeV}$,

$$
0.2 \times 10^{-31} \mathrm{~cm}^{2}<\sigma^{\nu N}<1.5 \times 10^{-31} \mathrm{~cm}^{2} .
$$

Thus, the CCNC correction to the massless $\sigma^{\nu N}$ is comparable with $\sigma^{\nu N}$.

At small Bjorken $x$ the unitarity/saturation effects enter the game $[13,14]$. Corresponding correction to $\sigma^{\nu N}$ was estimated in [5] as a 50 per cent effect. Particularly, it was found that the account of the unitarity turns the charged current cross section

$$
\sigma^{\nu N} \simeq 1 . \times 10^{-31} \mathrm{~cm}^{2}
$$


at $E_{\nu}=10^{12} \mathrm{GeV}$ into

$$
\sigma^{\nu N} \simeq 0.5 \times 10^{-31} \mathrm{~cm}^{2}
$$

In [5] the massless quark approximation was used. The unitarity effect is known to depend on the hardness scale of the process. The first higher twist correction is usually estimated as $[15]$

$$
\sim \frac{\alpha_{S}\left(Q^{2}\right)}{Q^{2}} \frac{G\left(x, Q^{2}\right)}{\pi R^{2}} .
$$

It was noted above that the CCNC hardness scale is much "harder" than the corresponding scale for light flavors: $m_{t}^{2} \gg Q^{2} \sim m_{W}^{2}$. Therefore, the unitarity correction to the CCNC component of $\sigma^{\nu N}$ is expected to be much smaller.

Summarizing, it is shown that in the UHE neutrino interactions the higher twist corrections brought about by the non-conservation of the top-bottom current dramatically change the longitudinal structure function, $F_{L}$, Eqs.(14). We started with the $\boldsymbol{\kappa}$-factorization formula for the differential cross section $d \sigma_{L} / d z d^{2} \mathbf{k}$ and derived simple and numerically accurate DLLA expression for $F_{L}$. It is worth emphasizing that the appearance of the factor $m_{t}^{2} /\left(m_{t}^{2}+Q^{2}\right)$ in (14) is not a property of the interaction of $t \bar{b}$-dipole with the target but the property of the light-cone density of $t \bar{b}$-states [8]. Only relative smallness of $Q^{2}$ restricted by Eq.(1) prevents the contribution of the CCNC term to $\sigma^{\nu N}$ from vanishing. The rapidly rising gluon density factor provides its additional enhancement. We neglected here the contribution of hard gluons to $F_{L}$. Therefore, the DLLA gives the lower estimate for the CCNC contribution to $\sigma^{\nu N}$. This contribution appears to be numerically large and comparable with $\sigma^{\nu N}$ evaluated in the massless quark approximation. Curiously, the CCNC effect in its competition with massless calculations gains momentum also from the unitarity suppression which is much stronger for the massless component of $\sigma^{\nu N}$.

Acknowledgments. Thanks are due to G. Ciapetta for the help in preparation of the manuscript. V.R. Z. thanks B.G. Zakharov for discussions and the Dipartimento di Fisica dell'Università della Calabria and the Istituto Nazionale di Fisica Nucleare - gruppo collegato di Cosenza for their warm hospitality while a part of this work was done. The work was supported in part by the Ministero Italiano dell'Istruzione, dell'Università e della Ricerca and by the RFBR grant 09-02-00732. 


\section{References}

[1] J.K. Becker, Phys. Reports, 458, 173 (2008).

[2] G.M. Frichter, D.W. McKay and J.P. Ralston, Phys. Rev. Lett. 74, 1508 (1995); Erratum-ibid. 77, 4107 (1996); R. Gandhi, C. Quigg, M.H. Reno and I. Sarcevic, Astropart. Phys. 5, 81 (1996); Phys. Rev. D 58, 093009 (1998); G. Parente and E. Zas, Proc. of the 7th Intern. Symp. on Neutrino Telescopes, Venice, Italy, Feb. 1996, p. 499 (astro-ph/9606091); G.C. Hill, Astropart. Phys. 6215 (1997); M.V.T. Machado, Phys.Rev. D70, 053008 (2004); R. Fiore, L.L. Jenkovszky, A.V. Kotikov, F. Paccanoni and A. Papa, Phys.Rev. D73, 053012 (2006);

[3] M. Glück, S. Kretzer and E. Reya, Astropart.Phys. 11, 327 (1999); J. Kwiecinski, Alan D. Martin and A.M. Stasto, Phys.Rev. D59, 093002 (1999); Yu Seon Jeong and Mary Hall Reno, Phys.Rev. D81, 114012 (2010); M. Glück, P. Jimenez-Delgado and E. Reya, Phys.Rev. D81, 097501 (2010).

[4] E.M. Henley and J. Jalilian-Marian, Phys.Rev. D73, 094004 (2006).

[5] K. Kutak, J. Kwiecinski, Eur.Phys.J. C29, 521 (2003).

[6] R. Fiore and V.R. Zoller "UHE neutrinos: current non-conservation, mass scales, saturation" talk at Diffraction 2010, Otranto (Lecce), Italy, September 10 - 15, 2010.

[7] V. Barone, M. Genovese, Nikolai N. Nikolaev, E. Predazzi and B. Zakharov, Phys.Lett. B328, 143 (1994).

[8] R. Fiore and V.R. Zoller, JETP Lett. 87, 524 (2008); "Full of charm neutrino DIS", in '08 QCD and High Energy Interactions, Proc. of 43rd Rencontres de Moriond on QCD and Hadronic Interactions, La Thuile, Italy, 2008, e-Print: arXiv:0805.2090.

[9] R. Fiore and V.R. Zoller, Phys.Lett. B681, 32(2009); "Current non-conservation effects in $\nu$ DIS diffraction" in AIP Conf.Proc. 1105, 304 (2009).

[10] V.N. Gribov and L.N. Lipatov, Sov. J. Nucl. Phys. 15, 438 (1972); L.N.Lipatov, Sov. J. Nucl. Phys. 20, 181 (1974); Yu.L. Dkshitzer, Sov. Phys. JETP 46, 641 (1977); G. 
Altarelli and G. Parisi, Nucl. Phys. B126, 298 (1977); R.G. Roberts, The structure of the proton. (Cambridge Univ. Press, 1990)

[11] I.P. Ivanov and N.N. Nikolaev, Phys. Rev. D65, 054004 (2003).

[12] M. Glück, E. Reya and A.Vogt, Eur. Phys. J. C5, 461 (1998).

[13] O.V. Kancheli, Sov. Phys. JETP Lett 18, 274 (1973).

[14] N.N. Nikolaev and V.I. Zakharov, Phys. Lett. B55, 397 (1975); Sov. J. Nucl. Phys. 21, $227(1975)$

[15] L.V. Gribov, E.M. Levin, M.G. Ryskin, Phys. Rep. 100, 1 (1981); A.H. Mueller, J. Qiu, Nucl. Phys. B 268, 427 (1986). 